\title{
Comparison of the tonic stretch reflex in athetotic patients during rest and voluntary activity
}

\author{
PETER D. NEILSON ${ }^{1}$ AND C. J. ANDREWS ${ }^{2}$ \\ From the Division of Neurology, The Prince Henry Hospital and the Schools of Medicine and Physics, \\ University of New South Wales, Sydney, Australia
}

SUMMARY An electromyographic technique was used to record the tonic stretch reflex (TSR) responses to passive sinusoidal stretching of biceps brachii muscle in athetotic patients. The TSR transmission characteristics measured while the patient was in a relaxed state were compared with those measured while the patient was voluntarily active. The resting TSR and action TSR were found to differ markedly. Sensitivity, pattern, duration, and timing of the TSR responses were all changed during voluntary activity. The results suggest that the TSR in athetosis is functionally reorganized by supraspinal influences during voluntary activity. It is proposed that athetoid movements and action tremor may be a manifestation of abnormal action TSR caused by faulty supraspinal control during activity.

Athetosis has been described as a manifestation of fluctuating rigido-spasticity (Narabayashi, Nagahata, Nagao, and Shimazu, 1965). They described a neurophysiological analysis based primarily on electromyography. The limb was passively moved while the electromyogram (EMG) of the reflex response was recorded on an ink writing oscillograph. Rigidity was characterized by a reflex discharge continuing for more than $60 \mathrm{sec}$ while the muscle was kept in a stretched position. Spasticity exhibited a phasic pattern with EMG activity appearing only during the stretching movement. In athetosis the static phase of the stretch reflex was associated with a fluctuating abnormal contraction and in some cases a coexisting spasticity was observed. A similar electromyographic technique was used by Andrews, Neilson, and Knowles (1972) to assess the tonic stretch reflex (TSR) in athetotic patients who were participating in a drug trial to determine the influence of phenoxybenzamine on athetosis. They concluded that the involuntary movements of athetosis were an entity distinct from rigido-spasticity because patients with severe athetoid movements commonly had only mild rigido-spasticity and the pattern of in-

1 Centre Industries Research Scholar.

2 Edwin and Daisy Street Research Fellow in Neurology. voluntary movement was not altered when the rigido-spasticity diminished during treatment with phenoxybenzamine.

The TSR of the biceps brachii muscle in normal man is activated during voluntary contraction and the transmission characteristics of the TSR during voluntary action have been shown to be more complex than measured previously in anaesthetized or decerebrate cats or in spinal man, probably because of activation of long loop reflex pathways (Neilson, 1972). The concept of reflex transmission being functionally reorganized by supraspinal control to subserve complex movement has been discussed by Hongo, Jankowska, and Lundberg (1969). If stretch reflexes in athetosis are functionally reorganized during voluntary activity, electromyographic assessment of the TSR by passive stretching of muscles while the patient is resting may bear little relation to the reflex control of muscle during locomotion or voluntary movement.

The present study compares the characteristics of the TSR in athetotic patients when elicited by passive movement during the relaxed state and when elicited by passive movement during voluntary activity. Thus the influence of supraspinal pathways on spinal segmental mechan547 


\section{METHOD}

Ten cerebral palsied patients, brain damaged at birth, aged 22 to 41 years and presenting a clinical picture of athetosis, were tested. The TSR of biceps was measured using two different methods. The first method measured the TSR while the patient was at rest and so, for the purpose of this paper, will be called the resting TSR. The electromyographic method used to assess the resting TSR has been described in detail elsewhere (Burke, Gillies, and Lance, 1970; Burke, Andrews, and Gillies, 1971). The second method, which has been described previously by Neilson (1972), measured the TSR during voluntary activity and so will be called the action TSR.

RESTING TONIC STRETCH REFLEX (RESTING TSR) The EMG of biceps and triceps muscles were recorded via surface electrodes $5 \mathrm{~cm}$ apart over the bellies of the muscles. The elbow joint angle was recorded by a goniometer strapped to the arm. Voltage output from the goniometer was differentiated (time constant $-5 \mathrm{msec}$ ) to give a voltage proportional to the angular velocity. EMG, joint angle, and velocity of stretch were recorded on a Grass four channel polygraph and on an Offner type $S$ dynagraph.

The patient lay supine on a couch and was instructed to relax. The arm was moved passively about the elbow by the experimenter. Sinusoidal stretching movements were used to determine the sensitivity and timing of the reflexes. The joint angle was moved through a $90^{\circ}$ range with a sinusoidal motion having a frequency of $1 \mathrm{~Hz}$. A second sinusoidal test was performed on three patients. The limb was oscillated through a $30^{\circ}$ joint angle range about an average extended position and again about an average flexed position. In this way the influence of the mean muscle length on the resting TSR was studied.

The average reflex response to sinusoidal stretching was also recorded. The EMG from both biceps and triceps muscles was full-wave rectified and averaged in an ND801 Enhancetron. The sweep of the Enhancetron was triggered at the same point in each sinusoidal stretching cycle by the voltage output from the goniometer. The EMGs of the resting TSR response of 20 sinusoidal stretching cycles were averaged. The average response was displayed on a Tektronix 531A oscilloscope and photographed with a Polaroid Land C4 camera.

Timing of the resting TSR response was measured with respect to the sinusoidal stretching cycle. The point of maximum EMG activity was related to the corresponding phase angle in the sinusoidal stretching cycle. The reference or $0^{\circ}$ phase angle was taken as the most stretched position of the muscle during the sinusoidal stretching cycle.

The effect of reinforcing the resting TSR by clenching the fist on the side opposite to the limb being tested was investigated in all 10 patients. The sinusoidal stretching tests were repeated with the patient clenching the fist. An EMG of wrist flexors was used to indicate the degree of fist clenching.

ACTION TONIC STRETCH REFLEX (ACTION TSR) The $\overrightarrow{\vec{F}}$ patient lay supine on a couch with the right arm strapped into a frame which constrained movement to flexion-extension about the elbow. The EMG and integrated EMG (IEMG) of the biceps brachii were recorded via surface electrodes attached $5 \mathrm{~cm}$ apart $\triangle$ over the belly of the muscle. The IEMG was cali- के brated by adjusting the amplifier sensitivity to pro- $\overrightarrow{0}$ duce a $25 \mathrm{~mm}$ pen deflection while the patient supported a $10 \mathrm{~kg}$ weight from a point on the wrist $30 \vec{\omega}$ $\mathrm{cm}$ from the axis of elbow rotation. A goniometer attached to the arm frame recorded elbow joint angle. The EMG, IEMG, and joint angle were recorded on a Grass four channel polygraph.

The patient was instructed to hold the arm as sti is as possible in a $90^{\circ}$ joint angle position in spite ब्ञ disturbance forces applied to the limb through $\frac{7}{8}$ 을 spring connected $30 \mathrm{~cm}$ from the elbow. Sinusoidal disturbance forces were applied by connecting the other end of the spring to an oscillating cantilever arm on a sinusoidal stretching machine which hos $\stackrel{5}{\rightarrow}$ been described elsewhere (Neilson, 1972). The free $\vec{\theta}$ quency of the sinusoidal stretching machine was $\omega$ varied through the range 0 to $5 \mathrm{~Hz}$. The patient was required to sustain a voluntary contraction of the biceps brachii because the spring was stretched to a tension of $5 \mathrm{~kg} \mathrm{wt}$. The level of voluntary contraction in the biceps could be increased further without $\frac{\circ}{\circ}$ changing the elbow angle by simultaneously con- $\varrho$ tracting flexor and extensor muscles. Changes in $\overline{\overrightarrow{0}}$ elbow joint angle caused by the disturbance force were taken as a measure of length changes of the biceps muscle and the IEMG signal from biceps provided a measure of the action TSR response.

A mathematical analysis of the data was required? since the EMG of the action TSR response was mixed with potentials due to the voluntary activity. As described previously (Neilson, 1972), a crosscorrelation analysis between elbow angle and IEMG signals enables the action TSR response to be separated from the voluntary activity. Reflex transmission $>$ characteristics between elbow angle changes and IEMG responses were described by gain and phase $N$ frequency response curves calculated by the spectral ${ }^{\circ}$ analysis technique described by Jenkins and Watts 0 (1968). The auto power spectra of elbow angle and 
IEMG signals were calculated so that harmonic distortion could be identified.

The influence of the average length of the biceps muscle on transmission of the action TSR was assessed by repeating the tests with the patient holding the arm steady in a flexed position and then again in an extended position.

\section{RESULTS}

RESTING TONIC STRETCH REFLEX (RESTING TSR) Passive sinusoidal movement of the limb through a $90^{\circ}$ joint angle produced an EMG reflex response in the biceps muscle in all patients tested. The EMG of the resting TSR response consisted of a burst of activity at one point in the stretching cycle with negligible activity during the rest of the cycle (Fig. 1). One patient also displayed a second burst of EMG activity during shortening. Timing of the EMG burst relative to the sinusoidal stretching movement varied from test to test but always occurred during the early part of the stretch-that is, $110^{\circ}-170^{\circ}$ phase in advance of the most stretched position in the sinusoidal cycle (Fig. 1).

The magnitude of the resting TSR response depended on the mean length of the muscle. The EMG burst was smaller when the arm was oscillated about a mean extended position than it was for oscillation about a mean flexed position (Fig. 2). The EMG of the resting TSR response was increased in magnitude when the patient 'reinforced' by clenching the fist on the opposite side. The shape and timing of the response were unchanged.

ACTION TONIC STRETCH REFLEX (ACTION TSR) Sinusoidal disturbance forces applied to the limb caused an oscillation of the elbow joint angle through a range of $10^{\circ}-20^{\circ}$ (Fig. 3). The auto power spectrum of the elbow angle signal

(a) VELOCITY

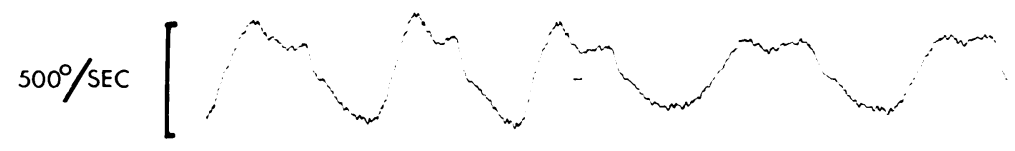

(b) ANGLE

EXT

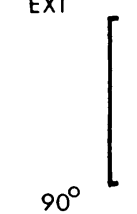

(c) EMG

I MV

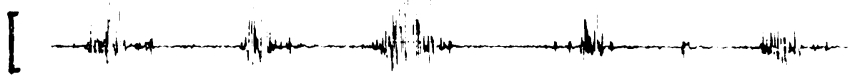

I SEC

FIG. 1. Resting TSR responses. Section of dynagraph recording showing typical EMG response patterns to sinusoidal stretching when patient is in a relaxed state. (a) Angular velocity of arm movement about elbow. (b) Elbow angle changes caused by passive sinusoidal movement. (c) EMG responses from biceps brachii muscle. The EMG response consists of a burst of activity early in the stretching movement with insignificant activity during the rest of the sinusoidal cycle. 


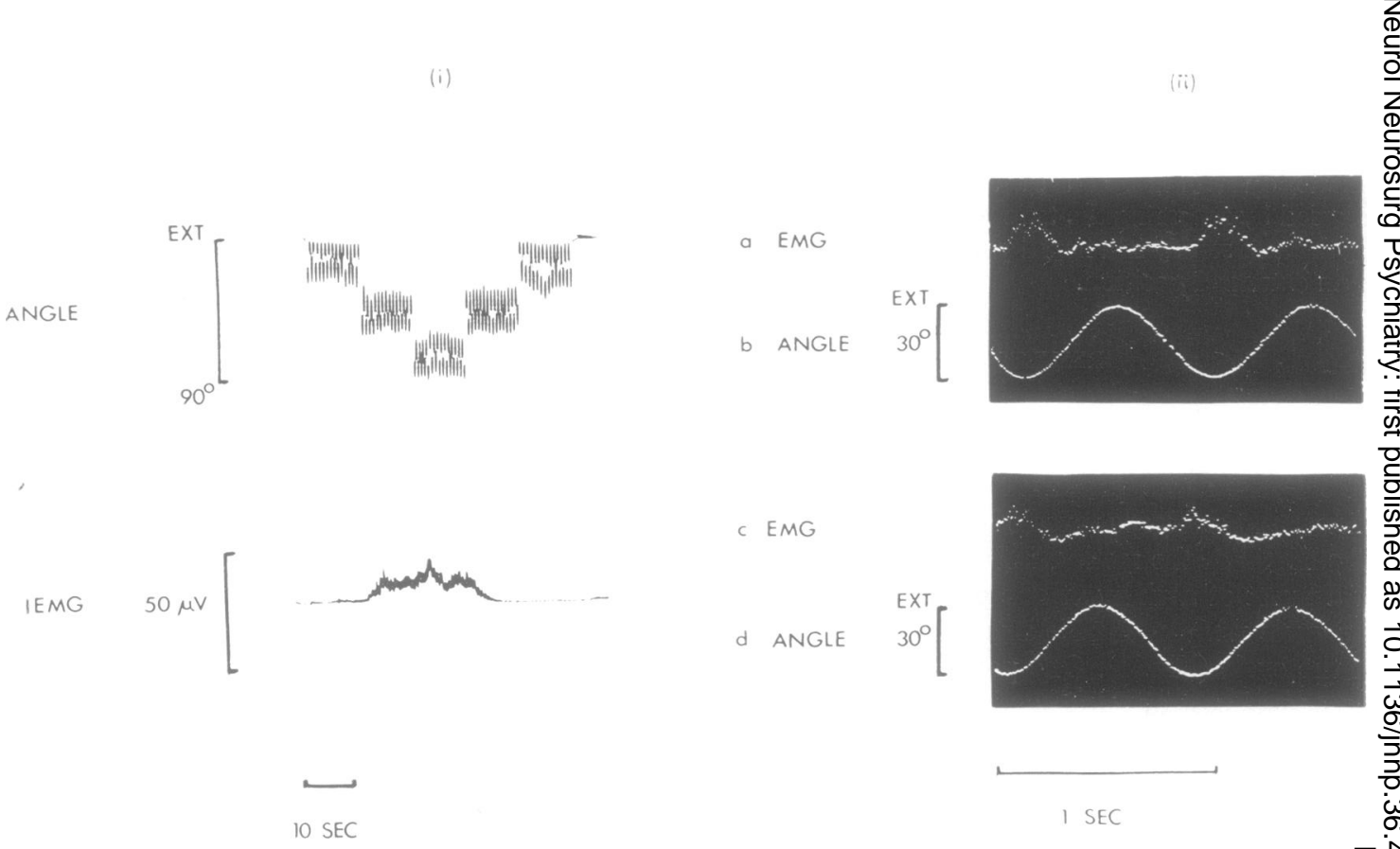

FIG. 2. Dependence of resting TSR on muscle length. (i) Dynagraph recording showing decrease in amplitu迹出 of the EMG response to sinusoidal stretch with an increase in the mean length of the muscle. a. Elbow angie

changes caused by passive sinusoidal movement. b. EMG responses of biceps brachii muscle.
(ii) Photograph of averaged EMG responses to sinusoidal stretching about different mean lengths of muscle. a. Average of 20 full wave rectified EMG responses from biceps brachii corresponding to joint ang changes shown in b. b. Averaged joint angle signals of 20 cycles of sinusoidal movement through a $30^{\circ}$ jogit angle about a mean flexed position. c. Average of 20 full wave rectified EMG responses from biceps brackita corresponding to joint angle changes shown in d. Notice averaged response is smaller than in a. d. Averagedo joint angle signals of 20 cycles of sinusoidal movement through a $30^{\circ}$ joint angle about a mean extended position.

showed a peak in the variance at the stretching frequency (Fig. 4). The absence of peaks at harmonics or sub-harmonics of the stretching frequency indicates that joint angle movement was sinusoidal with negligible harmonic distortion.

The EMG of the action TSR response was observed in all 10 patients tested but was mixed with potentials due to the sustained voluntary contraction (Fig. 3). The mathematical analysis which separated out the reflex responses from the voluntary activity produced the following power spectra. The auto power spectrum of the IEMG signal showed that the variance was distributed across the band of frequencies (0-5 $\mathrm{Hz}$ ) but there was a large peak in variance at the stretching frequency (Fig. 5). The cross power spectrum showed that the variance distributed across the band was due to random fluctuationso in the IEMG signal caused by the sustained产

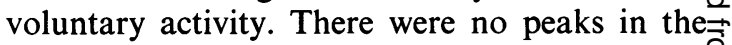
IEMG signal power spectrum at harmonics or 3 subharmonics of the stretching frequency (Fig. 5)? indicating that the IEMG of the action TSR? response was sinusoidal and therefore reflex transmission was linear, at least for the small? amplitude stretches tested. Reflex EMG activityo followed the full $360^{\circ}$ of the stretching cycle; it increased during stretch and decreased during shortening.

Timing of the action TSR response was mea-? sured with respect to the sinusoidal stretching $\frac{D}{0}$ cycle by calculating the phase shift between joint angle changes and IEMG changes. A cor- $N$ rection was made for the known phase lag intro- $N$ duced into the IEMG signal by the integrating N 
(a) ANGLE

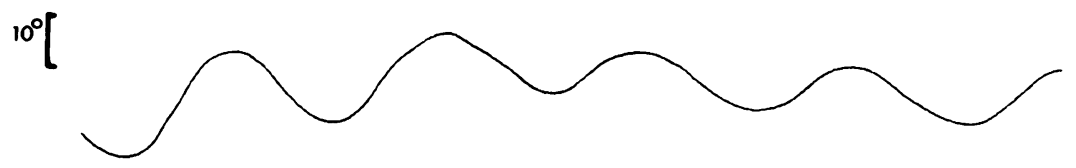

(b) EMG

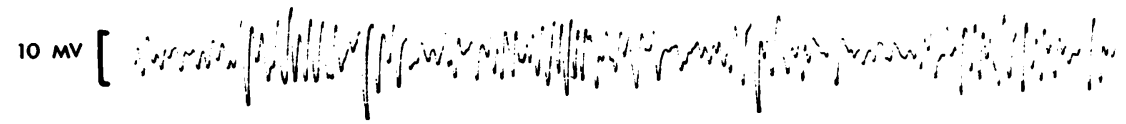

(c) IEMG

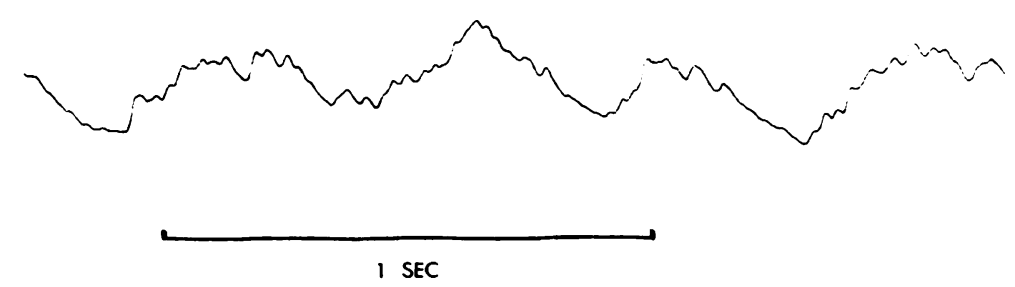

FIG. 3. Action TSR. Section of a polygraph recording showing sinusoidal elbow angle movements and typical EMG response patterns when the patient is sustaining a voluntary contraction of the biceps. (a) Sinusoidal elbow angle changes caused by disturbance force applied through spring. Extension is represented by a downward deflection. (b) EMG from biceps brachii. (c) IEMG (time constant $=0.16 \mathrm{sec}$ ) from biceps brachii. Notice that EMG responses are mixed with activity due to voluntary contraction, EMG responses follow full $360^{\circ}$ of sinusoidal stretch cycle, and maximum EMG occurs late in the stretching movement.

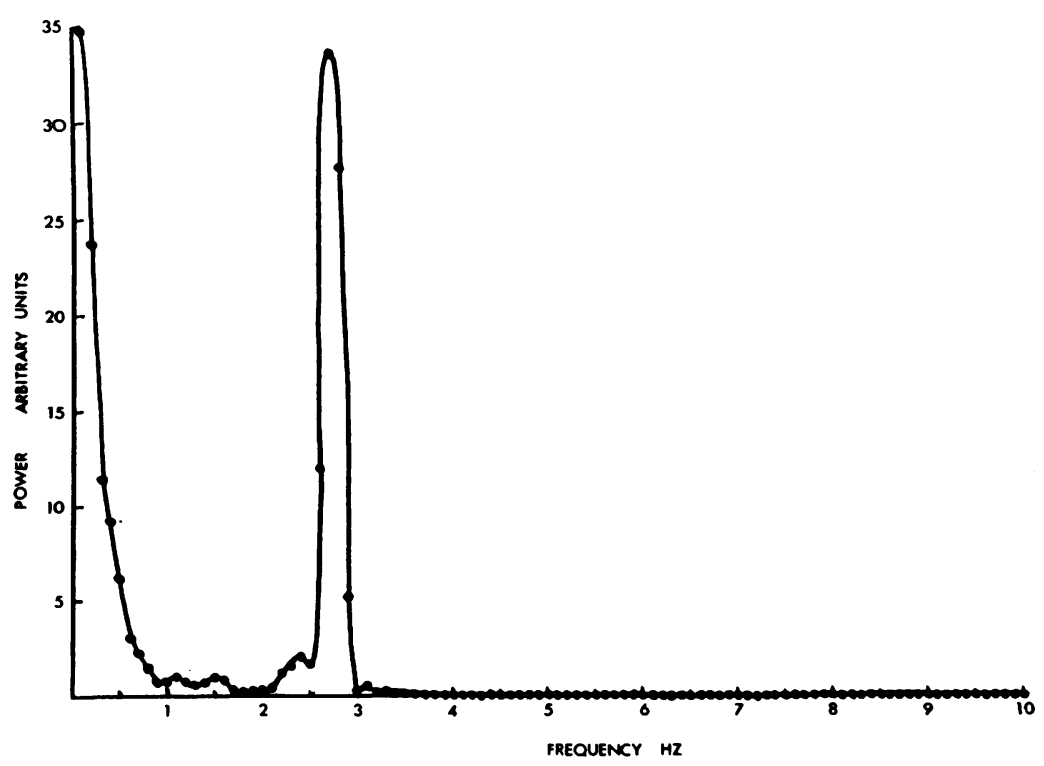

FIG. 4. Auto power spectrum of elbow angle. Auto power spectrum of changes in elbow angle caused by disturbance forces applied through spring. Power is plotted in arbitrary units proportional to elbow joint angle in degrees squared against frequency in $\mathrm{Hz}$. The spectrum shows the distribution of the variance of the joint angle signal with frequency. The peak at the driving frequency (2.7 $\mathrm{Hz}$ ) and the absence of peaks at harmonics of the driving frequency indicate that joint angle movement was a good approximation to a sinusoidal motion. 


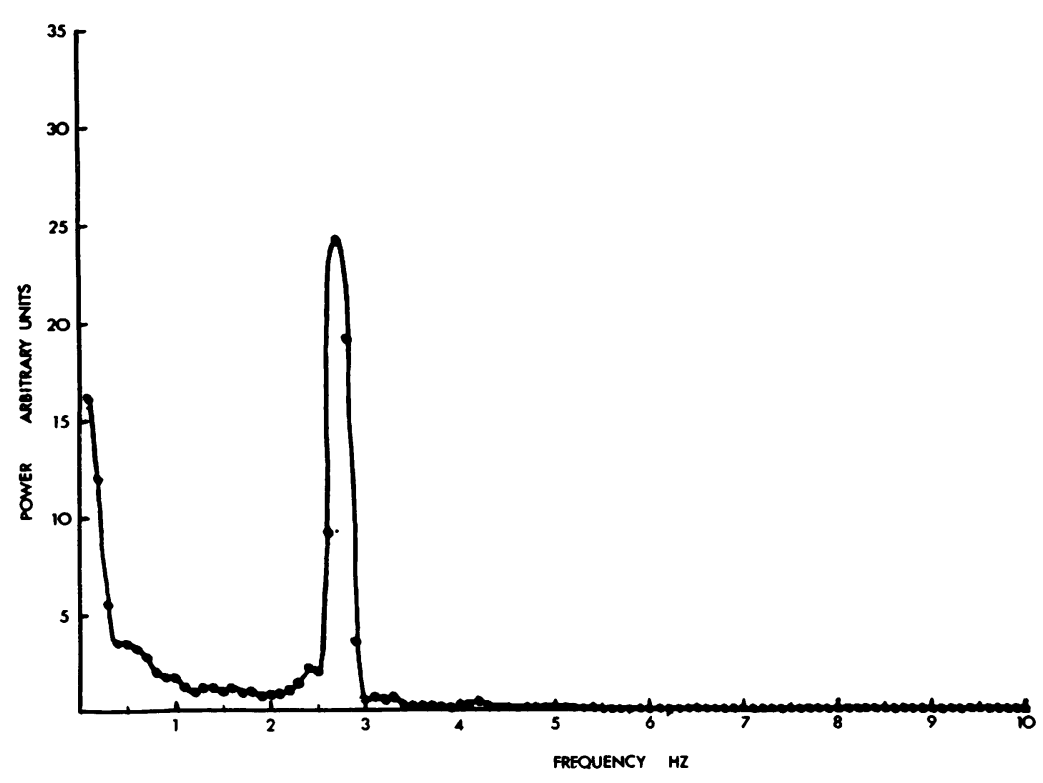

FIG. 5. Auto power spectrum of IEMG. Auto power spectrum of changes in the IEMG from biceps. Power is plotted in arbitrary units proportional to millimetres of pen deflection squared against frequency $\mathrm{Hz}$. The spectrum shows the distribution of the variance of the IEMG signal with frequency. Distribution of power across the band is due to random fluctuation in the IEMG signal caused by the voluntary activity. The peak at the stretching frequency $(2.7 \mathrm{~Hz})$ indicates: that the IEMG signal varied $\vec{\omega}$ predominantly at the stretching frequency. The absence of peaks at harmonics of the stretching frequency indicates that th⿻ action TSR response was of good approximation to a sinusoid.

filter. The EMG of the action TSR response had a phase lead of $10^{\circ}-50^{\circ}$ ahead of elbow angle changes.

No change in sensitivity or timing of the action TSR response was caused by alteration of the mean length of the muscle. Gain and phase characteristics were unchanged when the patient held the arm steady in a flexed position instead of an extended position.

COMPARISON OF RESTING TSR AND ACTION TSR Action TSR responses were sufficiently large to cause a deflection in the IEMG recording comparable with that produced when the patient supported $5-10 \mathrm{~kg} \mathrm{wt}$ at the wrist. The limb was stabilized in spite of sinusoidal disturbance forces of 5-10 kg wt. EMG responses of this order were not recorded in any patient during elicitation of the resting TSR. Although the EMG responses were difficult to quantify it can be stated that the action TSR was many times more sensitive to stretch than was the resting TSR. Large action TSR responses were measured to $10^{\circ}-20^{\circ}$ joint angle oscillations whereas $30^{\circ}-$ $90^{\circ}$ oscillations were required to produce reto tively small resting TSR responses.

The EMG of the action TSR response was not only larger than that of the resting TSR response but it had a completely different pattern. The IEMG of the action TSR was sinusoidal, it fol-o lowed the full $360^{\circ}$ of the stretching cycle. Transmission in the resting TSR on the other hand $\stackrel{\mathbb{Q}}{2}$ was non-linear, producing a burst of EMG at $\overrightarrow{\overrightarrow{0}}$ one point in the stretching cycle.

The timing of the action TSR was also different from that of the resting TSR. During voluntary activity the maximal amplitude of the EMG response occurred $10^{\circ}-50^{\circ}$ phase in advance of the position of maximal stretch, whereas, when 3 the patient was relaxed, the maximum of the EMG of the resting TSR response occurred at a point in the stretching cycle $110^{\circ}-170^{\circ}$ phase in advance of the position of maximal stretch. Since the stretching frequency was $1.0 \mathrm{~Hz}$, this phase difference corresponded to a $0.25-0.5 \sec N$ difference in timing.

The magnitude and timing of the action TSR $\tilde{D}$ response were unaltered by changes in the mean $\mathrm{\omega}$ 
length of the muscle. No difference in TSR transmission was observed when the patient held the arm steady in an extended position or a flexed position. On the other hand the resting TSR was found to be non-linear in this respect; sensitivity to stretch decreased as the mean length of the muscle was increased.

\section{DISCUSSION}

These experiments demonstrate clearly that the TSR of biceps brachii in athetotic patients was functionally reorganized by suprasegmental influences during voluntary activity. The EMG of the action TSR response was altered in most respects; the size, pattern, duration, and timing of the response were all changed.

Resting TSR transmission was non-linear because the burst discharge in the EMG of the response to sinusoidal stretching was nonsinusoidal and because the magnitude of the response altered with the mean length of the muscle. The burst in the EMG of the resting TSR cannot simply be attributed to a threshold phenomenon due to low excitability of alpha motoneurones under resting conditions because the timing of the response changed markedly during voluntary activity. A change in alpha motoneurone excitability could cause a burst discharge in the EMG by responding only during the period of maximum excitation in the sinusoidal cycle but it cannot explain the $0.25-0.5$ sec change in the timing of the TSR response which occurred during voluntary activity. The appearance of the burst in the EMG of the resting TSR at the beginning of the stretching cycle $\left(110^{\circ}-170^{\circ}\right.$ phase in advance of maximum stretch) could be explained by inhibition dependent on group II afferent nerve fibres (Burke et al., 1970), however, the non-sinusoidal response and the change of sensitivity with mean length suggest that other explanations based on nonlinear mechanisms are also possible. In contrast with resting TSR transmission, action TSR transmission was linear, the EMG response was sinusoidal and contained negligible harmonic distortions and neither gain nor phase altered with changes in the mean length of the muscle.

Transformation of TSR transmission characteristics during voluntary activity is consistent with the concept of supraspinal control functionally reorganizing the TSR to subserve voluntary movements. Interneurones are probably biased on during voluntary activity and then participate in reflex transmission so that different pathways are involved. This reorganization of pathways is probably responsible for the change in the TSR transmission characteristics.

It is not known whether involuntary movement and tremor in athetosis are caused primarily by lesions in the basal ganglia and other higher centres or whether they are secondary to other manifestations such as rigido-spasticity. Narabayashi et al. (1965) concluded that involuntary movement was secondary to fluctuating rigido-spasticity while Andrews et al. (1972) concluded that it was an entity independent of rigido-spasticity. The electromyographic technique used by both groups of authors measured rigido-spasticity while the patient was in a relaxed state. Since TSR transmission characteristics are functionally reorganized during voluntary activity, this may resolve the contradiction between the above views. Athetotic movement might be a manifestation of abnormal action TSR so that the resting TSR could be changed by phenoxybenzamine independently of involuntary movement and tremor. The hypothesis that athetotic movement and tremor may be a consequence of abnormal action TSRs caused by faulty supraspinal control is attractive because it suggests a physiological mechanism which explains the interaction between tremor, athetotic movement, and voluntary activity. It is well known that tremor and involuntary movement are exaggerated during voluntary activity and in many cases can only be observed when the patient is active.

Passive movement of the limb through a range at the joint and estimating whether or not the resistance to movement is increased is the standard method of assessing muscle tone at the bedside. The realization that the TSR in athetosis is functionally reorganized by supraspinal control during voluntary activity raises the question of the importance of clinical assessment of the action TSR. Is the action TSR in athetosis different from that in normals or in patients with other motor disorders? If there are differences are they involved in causing involuntary move- 
ment and tremor? Clearly these are important questions which must be the subject of future research studies.

\section{REFERENCES}

Andrews, C. J., Neilson, P. D., and Knowles, L. (1972). An electromyographic study of the rigido-spasticity of athetosis. Journal of Neurology, Neurosurgery, and Psychiatry, 36, 94-103.

Burke, D., Andrews, C. J., and Gillies, J. D. (1971). The reflex response to sinusoidal stretching in spastic man. Brain, 94, 455-470.

Burke, D., Gillies, J. D., and Lance, J. W. (1970). The quadriceps stretch reflex in human spasticity. Journal of Neurology, Neurosurgery, and Psychiatry, 33, 216-223.
Hongo, T., Jankowska, E., and Lundberg, A. (1969). The rubrospinal tract. 2. Facilitation of interneuronal transmission in reflex paths to motoneurones. Experimental Brain Research, 7, 365-391.

Jansen, J. K. S., and Rack, P. M. H. (1966). The reflex response to sinusoidal stretching of soleus in the decerebrate cat. Journal of Physiology, 183, 15-36.

Jenkins, G. M., and Watts, D. G. (1968). Spectral Analysi and its Applications. Holden-Day: San Francisco.

Narabayashi, H., Nagahata, M., Nagao, T., and Shimazu, H. (1965). A new classification of cerebral palsy based upon : neurophysiologic considerations. Confinia Neurologica, 25, 378-392.

Neilson, P. D. (1972). Frequency-response characteristics of the tonic stretch reflexes of biceps brachii muscle in intact man. Medical Biological Engineering, 10, 460-472. 